\title{
An Extended Ageing Study of a Uranium Dioxide Layer on Uranium Metal using the EDS/XPP Method
}

\author{
C. Poulter ${ }^{1}$
}

1. AWE, Reading, Berkshire, United Kingdom

There is general agreement that the presence of an oxide layer on uranium metal mediates its interaction with hydrogen ${ }^{[1]}$. Consequently models of the initial stages of the uranium-hydrogen reaction have been developed in terms of the hydrogen transport properties and thickness distribution of this surface oxide $^{[2]}$. For the purposes of validation or quantification of such models, it is essential to have a good understanding of the way in which oxide films grow on this metal (as a function of oxidising conditions) and how the mean oxide thickness and the thickness distribution is dependant on both oxidising conditions and exposure time ${ }^{[2]}$.

This work describes the analysis of an air grown Uranium dioxide layer on depleted Uranium metal using Energy Dispersive Spectroscopy in combination with calculations of the oxide thickness from measured X-ray intensities using the XPP method. An analysis of the oxide distribution is described and the relationship between mean thickness and thickness standard deviation is also explored.

Initial values for composition and oxide layer thickness were entered into the XPP model and using this data the optimum analysis conditions were deduced from calculations of the relative statistical variation at different beam voltages ${ }^{[3]}$ and the intensity of the Oxygen $\mathrm{K} \alpha$ line at $0.523 \mathrm{eV}$ was used to calculate the oxide thickness. A reasonable agreement has been shown between high and low carbon samples over an extended time (156 days air exposure) and the relationship between the Log-Normal distribution standard deviation and mean oxide thickness is compared for both sets of samples. This aspect being true if the standard deviation is expressed as a percentage of the mean oxide thickness or as an absolute value.

This extended ageing study has demonstrated the EDS/XPP method to be viable for the measurement of the thickness and distribution of a simple air grown uranium dioxide on depleted uranium metal and the data obtained has been used to further refine theoretical models of the oxidation and reaction kinetics of Uranium. Further work has given an indication that the limit in terms of oxide layer thickness for the XPP model has now been reached for the uranium samples but this data is not considered in the current study. The EDS/XPP method is further being explored for the measurement of oxides on Plutonium metal. 


\section{References:}

[1] R. Arkush et al, J. Alloys Compds. 244 (1996), p. 197.

[2] Glascott J, Phil. Mag. 94 (2014), P. 221

[3] Statham P. J. IOP Conf Ser.Mater.Sci.Eng. 7( 2010), p. 2027.

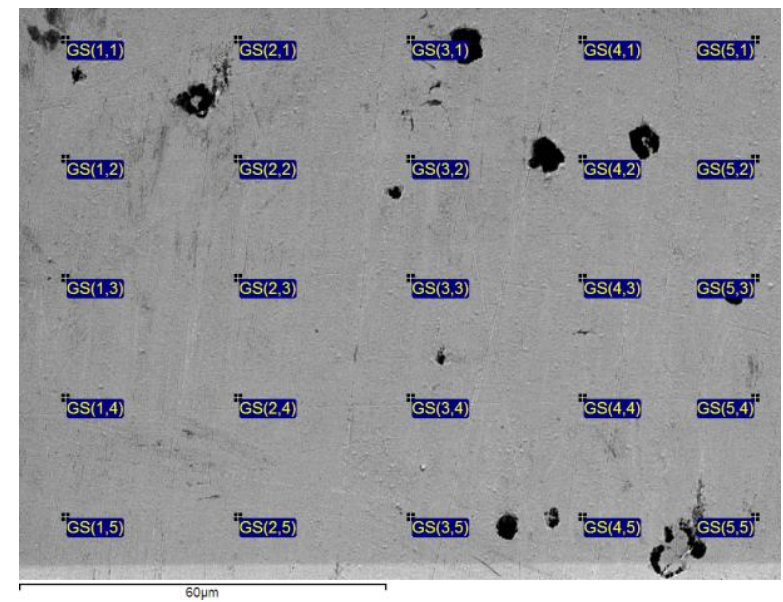

Figure 1. Showing a typical analysis area used for this study

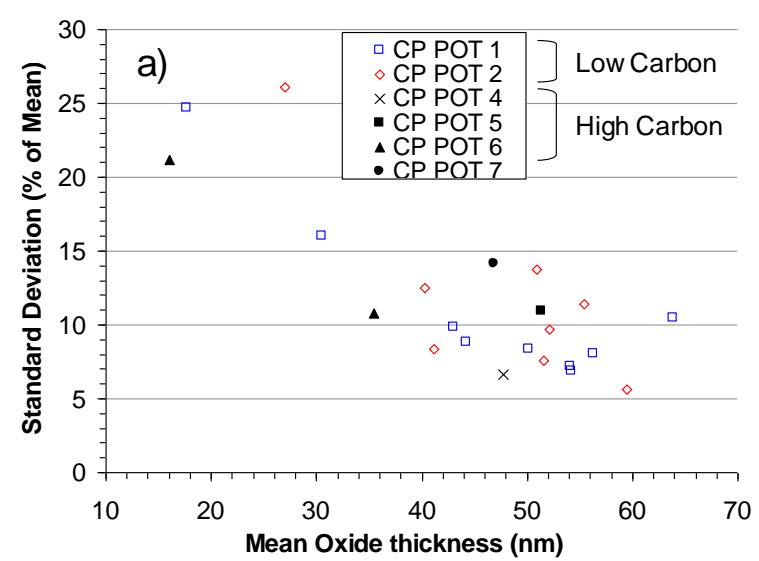

Figure 3. Standard deviation $\%$ of mean

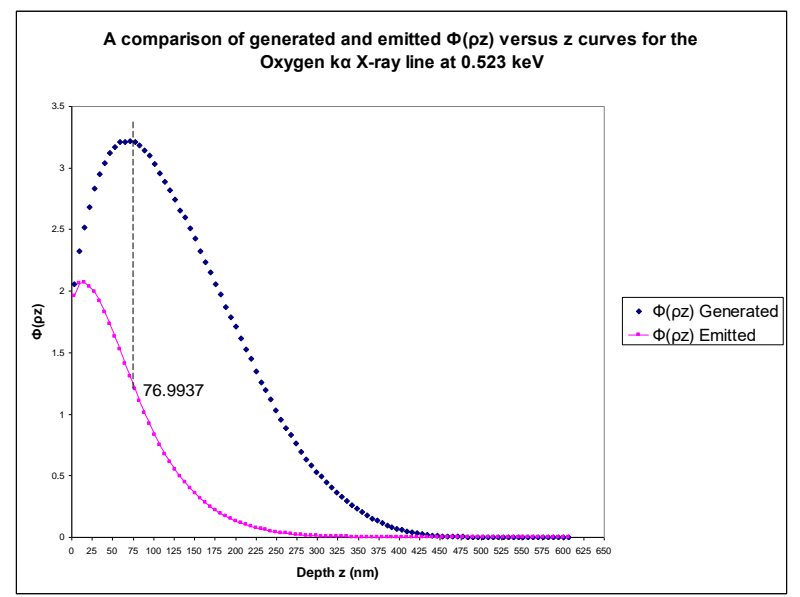

Figure 2. A comparison of generated and emitted $\Phi(\rho z)$ versus z curves for the Oxygen $k \alpha$ X-ray line

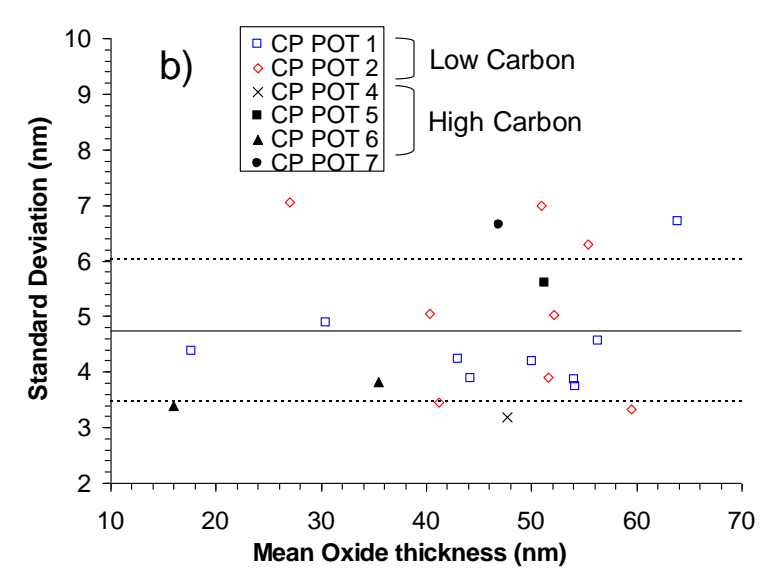

Figure 4. Standard deviation \% of thickness (nm) 